Revista de Investigación Educativa 9

julio-diciembre, 2009 | ISSN 1870-5308 | Xalapa, Veracruz

Instituto de Investigaciones en Educación | Universidad Veracruzana

\title{
Elementos de contexto para pensar la escuela
}

Jessica Badillo Guzmán

Instituto de Investigaciones en Educación

Universidad Veracruzana

El libro reseñado recupera los trabajos presentados en el Seminario Internacional Nuevos tiempos y temas en la agenda de política educativa. La escuela vista desde afuera, organizado por el IPPE/UNESCO, en el 2006. Los textos que lo integran buscan analizar los cambios que "desde afuera" (a nivel de contexto) están modificando la dinámica escolar y deberían ser tomados en cuenta en la formulación de las políticas en materia educativa.

Palabras clave: Educación, política educativa, contexto.

\section{Para citar este artículo:}

Badillo, J. (2009, julio-diciembre). Elementos de contexto para pensar la escuela. CPU-e, Revista de Investigación Educativa, 9. Recuperado el [fecha de consulta], de http://www.uv.mx/cpue/num9/ resena/badillo_pensar_escuela.html 


\section{Elementos de contexto para pensar la escuela}

Reseña. Tenti, E. (2008). Nuevos temas en la agenda de política educativa. Buenos Aires, Argentina: Siglo XXI editores.

\section{Introducción}

— xisten, en el campo de la educación, temáticas que han permanecido vi-

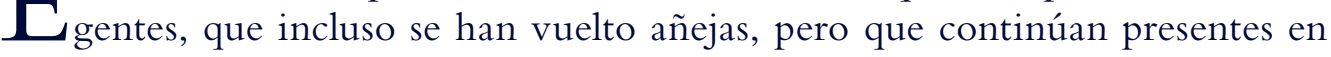
el discurso de la política educativa, en los planes y programas, tales como la calidad, los problemas en la cobertura y la necesidad de igualdad de oportunidades en el acceso a la educación. Pero por otro lado, hay otras temáticas que poco a poco se han ido imponiendo en el ámbito educativo, derivadas de las trasformaciones al interior del espacio escolar, pero también de los cambios en la vida social, en la estructura de las sociedades y en la forma de ser, pensar y actuar de sus actores.

En este sentido, el libro que Emilio Tenti nos ofrece representa una oportunidad de reflexionar la escuela de forma contextualizada. A lo largo de diez capítulos, nos encontramos con una serie de ensayos presentados en el seminario citado. En él se reunió a investigadores y funcionarios de América Latina y Europa con el propósito de discutir y reflexionar alrededor de los cambios contextuales y sus efectos en la educación.

De esta manera, se nos convoca a pensar la escuela poniendo atención en las nuevas dinámicas que desde los ámbitos económico, social, político, demográfico, están reconfigurando gradualmente la vida en la escuela. Así, "este libro busca contribuir a la mejor comprensión de algunas dimensiones de la vida social que necesariamente interactúan con el mundo escolar” (:I4).

\section{¿Desde dónde mirar la escuela?}

De acuerdo con Tenti, todos los cambios que se dan en la estructura social se manifiestan en las prácticas educativas y toman forma en sus actores. Así, hay una transformación innegable en la vida social que tiene fuertes repercusiones en la vida escolar, debido a las interacciones cada vez más complejas entre escuela 
y sociedad, que determinan su lógica pero que, al mismo tiempo, representan elementos a considerar en la construcción de la política educativa.

En este contexto, Tenti nos convoca a mirar la escuela desde afuera, esto es, considerando las nuevas características del entorno que vienen a tomar forma en el espacio escolar, pues como el autor afirma, "todo lo que sucede en la sociedad se siente en la escuela” (:I4). Así, las instituciones educativas ya no son lugares de élite, sino espacios donde se encuentra la riqueza con la exclusión social, con las nuevas culturas juveniles, en lo que el autor ha llamado "la invasión de la sociedad en la vida escolar".

Tenti aborda además un tema crucial: la brecha generacional entre estudiantes y profesores. Las nuevas identidades y las culturas juveniles se traducen en los modos de vivir la escuela y el profesor debería conocerlas más, para tener una mayor comprensión de sus alumnos, pues "sin esta comprensión, el trabajo y la interacción docente-alumno, que están en la base de la experiencia escolar, se vuelven fuente de conflictos, frustraciones y malestar para todos los involucrados” (:22).

\section{Nuevos temas}

Los autores que reúne el libro dan cuenta de las desigualdades entre las instituciones educativas en cuanto a infraestructura, financiamiento, recursos humanos, tiempos y modos de aprendizaje. Pero además, retoman las características de sus actores, los cambios en la juventud, en las familias y en sus condiciones socioeconómicas, la recomposición de los grupos sociales tradicionales y la emergencia de configuraciones culturales juveniles, cada una con sus propias demandas, intereses, valores y proyectos.

Por tanto, para discutir la escuela, para analizar sus prácticas y las líneas de política que alrededor y en ella se establecen, los autores nos acercan a estos nuevos temas, a reconocer que el afuera cambia, que la sociedad se ha revestido de nuevos valores, de nuevos procesos y nuevos actores; que la política, la distribución de la riqueza, las prioridades globales se han reconfigurado y que nuevos elementos se han incorporado.

En el Capítulo 1. El contexto de la política educativa, Daniel Filmus inicia reconociendo que los nuevos temas en la agenda de política educativa no son tan nuevos; en todo caso, lo que es nuevo es la perspectiva desde la cual se les analiza. 
Como Luis Aguilar plantea, el proceso de formación de las políticas públicas empieza por la formación de la agenda, esto es, "el conjunto de problemas, demandas, cuestiones, asuntos, que los gobernantes han seleccionado y ordenado como objetos de su acción, y más propiamente, como objetos sobre los que han decidido que tienen que actuar" (Aguilar, 2003:29). En el caso de las políticas educativas, este conjunto de problemas se ha venido heredando y lo que se hace más bien es replantearlos y reincorporarlos a la agenda, con unos cuantos avances y con muchos pendientes (en México, por ejemplo, los planes sexenales de educación constantemente vuelven sobre la cobertura y la calidad como parte de sus desafíos, de sus objetivos y metas).

Ante las variaciones en el contexto, Filmus remarca la necesidad de pensar la política educativa desde un proyecto de país, donde la justicia y la inclusión sean piezas clave. Para este autor, la escuela debe ser vista como parte del proyecto nacional, como una herramienta para su desarrollo. En este sentido, los "nuevos temas", podrían ser mejor abordados si se reconoce la importancia de la educación en el proyecto de país, no sólo a nivel de discurso, sino en la práctica.

Bernard Lahire, por su parte, en el Capítulo 2. Cultura escolar, desigualdades culturales y reproducción social, cuestiona la noción de Bourdieu de capital cultural y el uso que se le ha dado en la explicación de las diferencias sociales. Más aún, nos lleva a distinguir entre lo que constituye diferencia y lo que constituye desigualdad.

Así, para este autor, la desigualdad en el acceso a la educación sólo será considerada como tal cuando socialmente se le reconozca; mientras tanto, seguirá siendo una diferencia entre clases sociales, entre grupos, hasta cierto punto explicable en relación con su nivel de capital cultural.

Desde esta perspectiva, el autor llama a los sociólogos a dejar de medir sin reflexividad las distancias sociales a partir de un capital cultural y a tomar como objeto aquello que constituye un capital para los individuos y no desde una construcción teórica.

En el Capítulo 3. ¿Son posibles las políticas de subjetividad?, Juan Carlos Tedesco plantea el análisis de la subjetividad desde el punto de vista de las políticas educativas, teniendo en cuenta los cambios sociales, la crisis de cohesión social que prevalece en la actualidad y la necesidad de construir sujetos reflexivos en la escuela. El autor señala que es necesario reconocer el derecho que tenemos como individuos a la subjetividad, a desarrollar nuestra capacidad de elegir y ponerla en práctica, a construir nuestra propia identidad.

En este orden de ideas, nos llama a reflexionar sobre la ruptura de la cohesión social y sus impactos en la subjetividad; dicha ruptura está asociada a la des- 
igualdad, a la exclusión y a la marginalidad, contextos en los que los individuos no pueden desarrollar su capacidad subjetiva, pues sus posibilidades de decisión y de actuación son escasas. Este es un problema derivado de la vida social. Desde esta perspectiva, Tedesco recupera la pregunta de Touraine en cuanto a que si el fin de la sociedad puede conducir al nacimiento del sujeto, como una forma de sostener la necesidad de políticas de subjetividad, esto es, "políticas destinadas a garantizar condiciones institucionales que permitan cumplir con el derecho a la subjetividad para todos" (:6I).

Entre estas políticas de subjetividad, las que tienen que ver con la escuela deben partir del reconocimiento del estudiante como el centro de la acción educativa, enfocadas al desarrollo de sus potencialidades y capacidades. Esta cuestión debe plasmarse no sólo a nivel de discurso sino en las prácticas escolares, específicamente en la actividad del profesor.

En el Capítulo 4. Reconfiguraciones de la comunicación entre escuela y sociedad, Jesús Martín-Barbero recupera puntualmente una serie de elementos contextuales que están reconfigurando las prácticas escolares, pero también, la relación entre la escuela y la sociedad, donde ésta última está pasando de una sociedad con sistema educativo a una sociedad educativa.

Según este autor, vivimos una "era informacional", donde los espacios, los tiempos, las edades para aprender, son todos. El lugar privilegiado de la escuela como agente formativo ha quedado relegado por la Internet; el libro escrito es revelado de su centralidad ordenadora, pues poco a poco es sustituido por nuevos flujos de información, en una variedad exhaustiva de formatos.

Para Martín-Barbero, el sistema educativo vive una crisis provocada por cuatro cambios principales: I) la escuela y la familia ven erosionadas su capacidad educadora y su autoridad; 2) la escuela atraviesa una dificultad creciente para articular sus funciones de transmisión de la herencia cultural, capacitación y formación de ciudadanos; 3) la devaluación de la escuela pública; 4) la mutación tecnológica y sus repercusiones en la sociedad y en las aulas.

Aunado a lo anterior, surgen nuevos "modos de estar juntos": las familias se han reconfigurado, o en algunos casos, ya no existen; la noción de hegemonía del cuerpo nos ayuda a comprender el surgimiento de una cultura de los jóvenes, los tatuajes, los adornos, el enflaquecerse, todos ellos signos de una nueva forma de ser joven, aunada a la empatía tecnológica, desde la cual los jóvenes entran en contacto con múltiples oralidades y desde las cuales se comunican en nuevos idiomas, desconocidos en su mayoría para los adultos, indescifrables. 
Otro elemento fundamental que el autor aborda es la reconfiguración del mundo del trabajo y del mapa profesional, donde las identidades profesionales son transformadas y en su mayoría revestidas por la flexibilidad laboral, a la que el autor critica fuertemente, pues afirma que ésta acaba con la posibilidad de construir proyectos de vida vinculados con la actividad profesional que se realiza, ahora sólo importa poseer competencias; esto es, el trabajo se aleja de la concepción de una carrera profesional, soslayada por la competitividad y la flexibilidad laboral.

En este contexto de reconfiguración social y escolar, los Capítulos 5 y 6 nos introducen en los estudios sobre la juventud. Urresti, en el Capítulo 5. Nuevos procesos culturales, subjetividades adolescentes emergentes y experiencia escolar, nos adentra en la noción de subjetividad (tratada en el Capítulo 3 por Tedesco), entendida como identidad y reconociendo como sus ejes centrales a las narrativas y la experiencia histórica. Sobre esta base, el autor aborda algunas condiciones de época que tienen consecuencias importantes sobre la conformación de la subjetividad de las generaciones jóvenes. Así, distingue entre "adolescentes clásicos" y las nuevas formas y subjetividades juveniles, donde los adolescentes están cada vez más interesados en la moda, en la imagen y los jóvenes postergan la llegada de la adultez, en una clara tendencia a lo que el autor denomina "la juvenilización de la sociedad".

Según Urresti, estamos ante "neo-culturas juveniles" que enfatizan las opciones esteticistas, de rebelión simbólica, cuya proliferación es mucho más factible gracias a los usos que hacen los jóvenes de las nuevas tecnologías de la comunicación. Desde esta perspectiva, el autor reconoce que las nuevas culturas juveniles, como las antiguas, no se llevan muy bien con la autoridad de la escuela y, sin embargo, ésta sigue siendo el espacio donde pasan la mayor parte de su tiempo. Por tanto, afirma que la escuela no puede pasar por alto estas nuevas configuraciones culturales juveniles, sino más bien, repensarse a partir de ellas y de sus nuevas exigencias, no sólo en términos de la relación pedagógica, sino también en cuanto a sus estrategias, a los saberes y la forma en que los transmite.

El tema de las subjetividades juveniles tiene continuidad en el Capítulo 6. Instituciones desafiadas. Subjetividades juveniles: territorios en construcción, donde Rossana Reguillo nos brinda datos socioeconómicos importantes sobre la juventud, particularmente en el caso de México; pero además, retrata algunas de las características de la condición juvenil, entre las que destacan el desinterés, la incertidumbre, nada a largo plazo. Como García Canclini rescata en su texto Los jóvenes no se ven como el futuro: ¿serán el presente?, en la Encuesta Nacional de la Juventud 
2005 "el futuro es tan incierto que es mejor vivir al día fue la frase preferida por más de la mitad de los entrevistados" (García, 2008:5).

La autora identifica además tres ejes que juegan en la construcción identitaria juvenil: los procesos de informalización de las dinámicas juveniles, el repliegue del Estado social y el descrédito de las instituciones modernas.

Como ejemplo, Reguillo nos aproxima a tres subjetividades, a tres "biografías emergentes", mediante las cuales nos muestra que la identidad social no puede entenderse ni analizarse al margen de las condiciones objetivas que la hacen posible y que las posibilidades de construcción de la subjetividad juvenil son diversas.

El Capítulo 7, a cargo de Francoise Caillods, aborda el tema del fracaso escolar y las políticas más comunes para abatirlo. Así, el autor aborda los indicadores del fracaso escolar, entre los que figura el nivel de conocimientos alcanzado por los alumnos, el cual se ha convertido en uno de los referentes más importantes a partir de las pruebas estandarizadas internacionales.

Desde esta perspectiva, Caillods presenta los resultados obtenidos por algunos de los países pertenecientes a la OCDE, desde donde identifica a los sectores más vulnerables al fracaso escolar: en términos de género, los varones obtienen peores resultados escolares, de acuerdo con las pruebas PISA y PIRLS; los mismos estudios demuestran que los hijos e hijas de inmigrantes de primera o segunda generación tienen mayores dificultades de aprendizaje. Así también, los estudiantes que provienen de los sectores socioeconómicos más desfavorecidos y los provenientes de familias pobres obtienen malos resultados en las pruebas, además de aquellos que tienen dificultades de aprendizaje derivadas de problemas físicos o psicológicos.

En este tenor, el autor nos presenta algunas de las estrategias que se han instrumentado para luchar contra el fracaso escolar, entre las que destaca el fortalecimiento de la educación preescolar o maternal, algunos ejemplos de prácticas pedagógicas eficaces y el hecho de favorecer la heterogeneidad social en las escuelas, esto es, que los estudiantes se ayuden entre sí y evitar la segregación.

Caillods culmina con una serie de recomendaciones a partir de las experiencias exitosas en la lucha contra el fracaso escolar implementadas por los países desarrollados, las cuales considera que pueden ser un modelo para Latinoamérica.

El Capitulo 8. ¿El fin de la meritocracia?, de Agnes van Zanten, nos invita a reflexionar sobre los cambios recientes en las relaciones de la escuela con el sistema económico, político y social, entre los cuales destaca la evolución de la relación de la escuela con las diferentes categorías sociales. 
Para esta autora el poder meritocrático de la escuela se debilita en la medida en que la obtención de credenciales ya no garantiza movilidad social, recompensas profesionales, como lo hacía antes. Según van Zanten, la meritocracia es sustituida poco a poco por la "parentocracia", donde los padres de las clases sociales altas tienen mayor poder de decisión sobre la educación de sus hijos y la libertad de elegir las mejores escuelas, lejos de las clases sociales más desfavorecidas.

Luego de haber repasado una serie de elementos de contexto y algunas cuestiones de la dinámica escolar, Álvaro Marchesi, en el Capítulo 9, nos adentra en el análisis de los cambios sociales y la relación con las emociones y los valores de los docentes. Así, el autor recupera un aspecto que muchas veces pasa desapercibido por las instituciones, e incluso por los analistas: la condición psico-afectiva de los profesores. Al respecto, Marchesi afirma que los docentes han perdido la confianza en su actividad y que su autoestima, por lo tanto, ha bajado considerablemente, pues se sienten demasiado presionados por la sociedad, que ha desvalorizado su papel.

Así mismo, defiende la importancia de las emociones en la actividad docente, apuntando que cada vez más los profesores sufren de una tensión emocional derivada de los constantes cambios en el entorno y al interior de la escuela, los cuales definitivamente están afectando su práctica y su experiencia escolar. Para finalizar, el autor subraya la importancia del bienestar emocional y del cuidado que los mismos profesores deben tener de su estado emocional, pues éste repercute significativamente en su actuación y en los resultados educativos de sus estudiantes.

El Capítulo 10. De problemas a temas en la agenda de políticas educativas, es resultado de las reflexiones finales del Seminario Internacional del cual surge el libro. En él, Margarita Poggi enfatiza la importancia de las temáticas en la construcción de políticas educativas para el logro de una igualdad que sea capaz de recuperar y atender las diferencias sociales y culturales. Por otro lado, hace hincapié en la necesidad de estudiar los efectos de las políticas, más allá de su elaboración.

\section{Concluyendo}

El texto que se ha reseñado recoge las nuevas temáticas que emergen en el campo de la educación, particularmente en el ámbito de las políticas educativas, por lo que se convierte en un referente obligado para todos aquellos estudiosos de la escuela, de sus actores y de sus procesos. 
Ofrece al lector la posibilidad de pensar y repensar sus propias ideas en torno a la escuela y su papel en la sociedad, una sociedad que cambia, que influye en las prácticas educativas y en la cual emergen nuevas subjetividades que confluyen en los espacios educativos y que no pueden ser ignoradas ni homogeneizadas.

Es un texto para reflexionar, para discutir con y a partir de él, pero sobre todo, para entender que la escuela y la sociedad necesitan conocerse y reconocerse, pues sólo así podrán tender puentes que favorezcan una relación dialógica, renovada y renovadora.

\section{Lista de referencias}

Aguilar, L. F. (2003). Estudio Introductorio. En Aguilar, L. F. (Editor). Problemas públicos y agenda de gobierno. Colección Antologías de Política Pública. Tercera antología. México: Miguel Angel Porrúa.

García, N. (2008). Los jóvenes no se ven como el futuro: ¿serán el presente? En Pensamiento Iberoamericano. Núm. 3, segunda época.

Tenti, E. (2008). Nuevos temas en la agenda de política educativa. Buenos Aires, Argentina: Siglo XXI editores. 